\title{
Randomized Controlled Trial to Assess the Impact of Intraurethral Lidocaine on Urodynamic Voiding Parameters
}

\author{
Cassandra K. Kisby, MD ${ }^{1}$, Eric J. Gonzalez, $\mathrm{PhD}^{2}$, Anthony G. Visco, $\mathrm{MD}^{3}$, Cindy L. \\ Amundsen, $\mathbf{M D}^{3}$, and Warren M. Grill, $\mathrm{PhD}^{2}$ \\ ${ }^{1}$ Department of Obstetrics and Gynecology, Duke Hospital, Durham, NC \\ ${ }^{2}$ Department of Biomedical Engineering, Duke University, Durham, NC \\ ${ }^{3}$ Department of Obstetrics and Gynecology, Division of Female Pelvic Medicine and \\ Reconstructive Surgery, Duke Hospital, Durham, NC
}

\section{Abstract}

Objectives-Our objective was to determine whether intraurethral anesthesia decreases voiding efficiency (VE; voided volume/(voided volume + residual volume)) and impacts other urodynamic parameters in healthy female volunteers during urodynamic studies (UDS).

\begin{abstract}
Methods-This was a randomized double-blind placebo-controlled study of asymptomatic women 18-60 years. Subjects completed a Visual Analog Scale (VAS) and baseline questionnaires to assess pain and lower urinary tract symptoms, respectively. They performed an uninstrumented baseline uroflow followed by physiologic filling to $\geq 250 \mathrm{~mL}$. Subjects were randomized to receive $5 \mathrm{~mL}$ of intraurethral aqueous gel or $2 \%$ lidocaine gel and then underwent a second uninstrumented uroflow. They then completed complex cystometry, urethral pressure profilometry, and pressure flow studies.
\end{abstract}

\begin{abstract}
Results - 23 randomized subjects (12 placebo, 11 lidocaine) were included. Baseline uroflow VE was similar between the placebo and lidocaine groups. After study drug administration, VE was not different between groups $(89.3$ [85.9,93.9] vs. 89.5 [82.5,91.7], $\mathrm{p}=0.74)$. There were also no differences between groups in VAS scores, sensation during cystometry, maximum urethral closure pressure, or micturition parameters (maximum detrusor pressure (Pdet) and Pdet at maximum flow). The placebo group had a lower percentage of interrupted flow pattern ( $0 \%$ vs. $36 \%, \mathrm{p}=0.02)$ and a lower rate of increased electromyographic (EMG) activity during micturition ( $25 \%$ vs. $73 \%, \mathrm{p}=0.02)$.
\end{abstract}

Conclusions-In this pilot study of 23 asymptomatic women, intraurethral administration of lidocaine did not decrease VE compared to placebo. The lidocaine group had a greater percentage of interrupted flow patterns and increased EMG activity during micturition.

\section{Keywords}

voiding efficiency; urethral anesthesia; urodynamics

Corresponding author: Cassandra K. Kisby, MD, Department of Obstetrics and Gynecology, Duke Hospital, 200 Trent Drive, Baker House 236, Durham, NC 27710, Cassandra.kisby @ duke.edu, (704) 813-1160.

Clinical Trials Registry: clinicaltrials.gov (NCT02823431) 


\section{INTRODUCTION}

The storage and elimination of urine is regulated by neural circuits in the brain and spinal cord to coordinate function between the urinary bladder and the urethra. ${ }^{1}$ During micturition, the elimination of urine is facilitated by bladder wall contraction and urethral wall and pelvic floor muscle relaxation. Animal studies also demonstrated that urine flow through the urethra activates sensory nerves to amplify bladder contractions and maintain efficient bladder emptying. ${ }^{2,3}$ In animals, the augmenting reflex is mediated by pudendal afferent nerves, ${ }^{8}$ and when these nerves are transected or blunted with anesthesia, animals demonstrated incomplete voiding and urinary retention.., 10 This augmenting reflex was also demonstrated through intraurethral electrical stimulation in both males and females with spinal cord injuries. ${ }^{4-7}$ However, the role of this reflex in voiding in healthy women is unknown.

Lower urinary tract symptoms, including incomplete bladder emptying, decrease quality of life, increase healthcare utilization, decrease workplace productivity, and impact intimate relationships. ${ }^{11,12}$ In women, therapies to improve incomplete emptying or urinary retention depend on the etiology and may include physical therapy, intermittent or indwelling catheterization, urethral dilation, pharmacotherapy, surgery, or neuromodulation. ${ }^{13}$ These treatments, however, do not address bladder dysfunction as the etiology of the retention and patients may develop overflow incontinence, hydroureter, hydronephrosis, infection, or renal failure. To improve therapeutic outcomes, there is need to clarify the neural mechanisms that contribute to urinary retention. The studies described herein compared urodynamic parameters in healthy women with or without urethral anesthesia to determine the role of urethral sensory feedback in efficient bladder emptying. We hypothesized that urethral afferent nerve fiber activation augments bladder contractions for efficient voiding and that intraurethral lidocaine would disrupt sensory feedback and impair bladder emptying.

The primary aims of this study were to (i) compare voiding efficiency (voided volume/ (voided volume + residual volume)) during uroflow in healthy female volunteers randomized to receive either intraurethral lidocaine or placebo, (ii) compare urodynamic parameters between the two groups, and (iii) evaluate whether urethral anesthesia impacted pain scores in women undergoing urodynamic studies (UDS).

\section{MATERIALS AND METHODS}

This was a randomized, double-blind, placebo-controlled trial of healthy women recruited at Duke University between October and December 2016. Approval for this study was obtained from the Duke University Health System Institutional Review Board and written informed consent was obtained from all participants. This trial was registered at clinicaltrials.gov (NCT02823431).

Prospective subjects were recruited via electronic and print advertisements and underwent an initial phone screen followed by an in-office screen to determine eligibility. The in-office screen consisted of a review of demographics, health history, and medications and completion of the validated Lower Urinary Tract Symptoms (LUTS) Questionnaire. ${ }^{14}$ 
Inclusion criteria were: (1) asymptomatic females ages 18-60, (2) able to provide informed consent and agree to the study risks, and (3) no health conditions as indicated in the exclusion criteria. Exclusion criteria were: (1) neurologic condition (Parkinson's disease, multiple sclerosis, myasthenia gravis, recent stroke), (2) interstitial cystitis or bladder pain syndrome, (3) recurrent urinary tract infections (UTI) or active UTI, (4) body mass index (BMI) $>40$ due to its associations with LUTS ${ }^{15}$ (5) $<6$ weeks postpartum or breastfeeding, (6) pelvic organ prolapse at or beyond the hymen, and (7) $>2$ replies of $\geq$ "sometimes" on the LUTS Questionnaire. Subjects were asked to abstain from caffeine and alcohol for 24 hours and medications with anticholinergic activity for 1 week prior to their study visit. Subjects were excluded if they were taking antimuscarinics for overactive bladder; anticholinergic medications that required a 1-week washout included antihistamines, psychiatric medications, and others listed on the Anticholinergic Risk Scale. Pregnant patients and those with $\geq 1+$ blood or dipstick urinalysis suspicious for UTI were also excluded.

Participants were randomly assigned to receive intraurethral application of $5 \mathrm{~mL}$ of plain aqueous gel (KY Jelly; Reckitt Benckiser, herein referred to as placebo gel) or 2\% lidocaine gel (Urojet; Amphastar Pharmaceuticals, El Monte, CA). The identically appearing study drug syringes were allocated into consecutively numbered opaque envelopes by an unmasked study coordinator who did not participate in study visits or analysis.

Randomization was performed after informed consent and determination of eligibility based on the in-office screen. MATLAB Version 9.0 (MathWorks Inc., Natick, MA) was used to create a balanced 26-item randomization scheme with equal numbers of placebo and lidocaine drug. Randomization envelopes were chosen consecutively by investigators (CK, EG) performing the UDS. The study was double-blind, as subjects and the research staff, aside from the unmasked coordinator, remained masked to study drug assignment for the duration of the study.

Following randomization, the subject's bladder was scanned transabdominally with a bladder scanner to ensure at least $250 \mathrm{~mL}$ was present. If adequate volume was not present, then more time was allowed for physiological filling. Once reaching our threshold capacity, the subjects completed a baseline uninstrumented uroflowmetry study and assessment of postvoid residual with a standard clinic bladder scanner. Subjects were asked to void in their usual fashion over a uroflowmetry load cell.

After baseline uroflowmetry, subjects were instructed to hydrate and return with a comfortably full bladder to complete standard UDS (Figure 1). ${ }^{16}$ When subjects returned with at least a $250 \mathrm{~mL}$ bladder capacity, we administered the randomly allocated study drug. Briefly, the urethral meatus was cleansed with betadine, the urethra was cannulated with an 18-gauge vascath tip attached to the syringe, and $5 \mathrm{~mL}$ of the drug was injected along the length of the urethra. For injection, the vascath tip was advanced along the length of the urethra and test injection was performed until some resistance was met. This resistance signified injection within the urethra; the remainder of the drug was injected distal to this point. The drug incubated for 3 minutes with the subject in a semi-reclined position. Standard voiding instructions were then reviewed with the subject and the subject underwent a second, post-drug uninstrumented uroflowmetry; uroflowmetry was performed around the 
5-minute point of incubation given the time needed for instruction and relocating the subject to the uroflow commode. The 3-5 minute incubation period was chosen based on the time of onset cited in the Amphaster Pharmaceuticals package insert. A postvoid residual was obtained both by bladder scanner and in and out catheterization.

After the second uroflowmetry, subjects completed complex cystometry, urethral pressure profilometry (UPP), and micturition pressure-flow studies. A dual-sensor 8-french catheter was inserted through the urethra into the bladder and an 8-french catheter was inserted into the vagina. Surface electromyography (EMG) pads were placed at the 3 and 9 o'clock positions on the perineum to assess pelvic floor muscle recruitment during voiding. After securing the leads to the patient, the bladder was filled with room-temperature normal saline at $60 \mathrm{~mL} / \mathrm{min}$. Standardized questions were administered to assess first sensation of filling, first desire to void, strong desire to void, and maximum cystometric capacity (MCC). The infusion pump was stopped at MCC. UPP was then performed by manually withdrawing the urethral catheter to measure urethral closure pressure. This catheter was re-inserted and the subject was asked to void into a beaker on the load cell with the pressure catheters in place (micturition pressure-flow study). A final PVR was obtained by in and out catheterization.

The Visual Analog Scale (VAS) is a continuous pain scale from 0-100 which has been previously validated for use in urogynecological research. ${ }^{17}$ It was administered by asking participants to place a hashmark on a $100 \mathrm{~mm}$ line during the following times: after baseline uroflow, after post-drug uroflow, following insertion of the urethral and vaginal catheters for complex cystometry, at $100 \mathrm{~mL}$ cystometric filling, and at MCC.

Sample size planning considered a single study of unanesthetized healthy controls who had a voiding efficiency of $93 \pm 0.1 \% .{ }^{18}$ However, there is a lack of data on the effect size of intraurethral lidocaine administration in women. We, therefore, used instead a convenience sample based on project feasibility. Our goal was to recruit 20 subjects.

Analysis was performed using SPSS Statistics for Macintosh, Version 24.0 (Armonk, NY: IBM Corp.). Continuous variables were analyzed by Student $t$ test and categorical variables were analyzed using Pearson's $\chi^{2}$ or Fisher Exact test. The Mann-Whitney U test was used where indicated for non-normally distributed data. $P$ values $₫ 0.05$ were considered statistically significant.

\section{RESULTS}

Forty-two women were recruited and 25 provided informed consent (Figure 2). One subject failed the LUTS questionnaire ( $>2$ replies of sometimes or more) and was excluded prior to randomization. Of the 24 randomized subjects, one subject was excluded due to paruresis. The final population for data analysis included 23 subjects randomized to the placebo $(n=12)$ and lidocaine $(\mathrm{n}=11)$ groups. The two study groups were overall similar in demographics and responses to baseline questionnaires, however there were trends toward more cesarean sections and vaginal deliveries in the placebo group and higher education in the lidocaine group (Table 1). Average flow rate, maximum flow rate, flow time, and voiding efficiency were similar on baseline uroflow between the two study populations (Table 2).

Female Pelvic Med Reconstr Surg. Author manuscript; available in PMC 2020 July 01. 
Our primary outcome, voiding efficiency on uroflow after administration of study drug, was not significantly different between placebo and lidocaine groups when assessed by either bladder scan or catheterization (Table 2). Average flow rate, maximum flow rate, and flow time were also unchanged on uroflow (Table 2).

Urinary bladder sensation, including first sensation of bladder filling, first desire to void, strong desire to void, and maximum cystometric capacity during cystometric filling were similar between placebo and lidocaine groups. In addition, maximum urethral closure pressure with urethral pressure profilometry was similar between study groups (Table 2). Idiopathic detrusor overactivity was unexpectedly observed during filling cystometry in one subject $(8 \%)$ in the placebo group and one subject (9\%) in the lidocaine group. Stress urinary incontinence and urge urinary incontinence occurred at maximum cystometric capacity in one subject with placebo instillation.

Pressure and flow measurements, including maximum detrusor pressure (Pdet), Pdet at maximum flow, maximum abdominal pressure during voiding, and flow time were not different between placebo and lidocaine groups during micturition studies (Table 2). Increased abdominal pressure (Pabd recruitment, as measured by the vaginal catheter) during voiding was more common in the lidocaine group (46\% vs. $8 \%$ ), although this was not statistically significant ( $\mathrm{p}=0.07$ ). Pelvic floor muscle EMG activity, however, was increased during voiding in the lidocaine group (73\%) compared to the placebo group (25\%) $(\mathrm{p}=0.02)$. Interrupted uroflow curve was observed in four subjects with lidocaine instillation, whereas all subjects with placebo had continuous urine flow patterns ( $36 \%$ vs. $0 \%, \mathrm{p}=0.02$ ). Post void residuals were similar between placebo and lidocaine groups and there were no differences in voiding efficiency when calculated using the micturition pressure-flow study (Table 2).

There were four subjects with abnormal baseline voiding characteristics, including PVR $>100 \mathrm{~mL}$, elevated baseline VAS score $(>50)$, or baseline uroflow $\mathrm{VE}<80$. Excluding these subjects with baseline voiding dysfunction, we found that voiding efficiency was lower in the lidocaine group ( $84 \%$ vs. $90 \%, \mathrm{p}=0.05$ ). It is unclear if this difference is clinically significant though it does demonstrate that there could be discrepancies in what patients report on a validated symptom questionnaire and objective voiding and sensory measurements on UDS.

After the baseline uroflow, Visual Analog Scale scores for pain were statistically similar between groups. The distribution of VAS scores following catheterization, at $100 \mathrm{~mL}$ of filling, and at maximum cystometric capacity were also unchanged between placebo and lidocaine groups (Table 3).

\section{DISCUSSION}

In this pilot study of healthy female volunteers, intraurethral $2 \%$ lidocaine gel did not decrease voiding efficiency during uroflow. However, a greater proportion of participants who received lidocaine demonstrated elevated EMG activity and an interrupted urinary flow pattern during pressure-flow studies. 
The majority of research on urethral anesthesia and blockade of the augmenting reflex has been conducted in animals. ${ }^{8,19,20}$ Peng et al. ${ }^{8}$ found that detrusor contraction amplitude and duration were lower following intraurethral infusion of $2 \%$ lidocaine in rats. As well, voiding efficiency was reduced from 63 to $23 \%(\mathrm{p}<0.05)$ in the lidocaine group and reduced by only $3.6 \%$ in the placebo group. It is notable that these animal studies used multiple cystometric fills as well as more invasive interventions including sensory nerve transection. Although these animal studies demonstrated large changes in voiding efficiency, the methods and conclusions are difficult to translate directly to the clinic.

Our study differs from prior studies that evaluated sensory feedback in humans. Shafik et al. 21 examined the effect of intraurethral 5\% xylocaine gel in males and females on micturition studies, after 15 minutes of drug incubation and instrumented cystometric filling. They reported increased strain-voiding, in the form of elevated vesical pressure. They noted elevated PVRs in the xylocaine group, but did not report VE. In the present study, we used an incubation time of 3-5 minutes for the $2 \%$ lidocaine, based on the manufacturer's estimated time of onset, and also because allowing physiologic filling meant participants returned for the second uroflow with a comfortable urgency to void. We also included only females, who have a shorter urethral length than males, thus smaller potential distance to which study drug may be applied. Finally, our primary outcome was voiding efficiency, as this is a clinically relevant and easy to measure clinical parameter.

In contrast to animal studies, we did not perform multiple cystometric fills to assess voiding efficiency but rather used routine urodynamic parameters during voiding following a single fill. In addition to the baseline and post-intervention uroflow studies to assess voiding efficiency, the current study included the micturition study to assess voiding. Although there was no difference in voiding efficiency between groups in our small sample, we found that a greater proportion of the subjects receiving lidocaine had elevated EMG activity and an interrupted urinary flow stream on micturition pressure-flow studies. Additionally, there was a trend toward having lower max flow (Qmax) rates and higher abdominal recruitment (Pabd) during voiding in the lidocaine group (Table 2). These findings suggest that participants are requiring more effort to empty their bladder. These subjective voiding patterns are similar to the strain-voiding observed in Shafik et al. ${ }^{21}$ It is unclear if comparing an objective biomarker such as voiding efficiency, which may have variance with repeated measures, or using subjective voiding patterns is more appropriate. Further studies should be performed to evaluate variance of voiding efficiency with repeated measures and also how well the objective measure of voiding efficiency versus subjective strain/valsalva voiding pattern correlates with clinically reported urinary retention.

Prior studies used varying techniques to administer intraurethral anesthesia including injection using a catheter in males or abutting clinically-designed applicators against the urethra. ${ }^{21,22}$ Chan et al. ${ }^{23}$ compared VAS scores during catheterization after application of $2 \%$ lignocaine or placebo lubricant in females and found a $15.4 \mathrm{~mm}$ reduction in pain with lignocaine application (13 mm difference is considered clinically significant ${ }^{24}$ ). Their method of injection was not completely delineated for both groups. We did not achieve this pain-reduction threshold at any time-point. For study drug application, we used a vascath tip attached to identical luer lock syringes. It is reasonable to assume this method, the 
concentration, or the agent chosen could influence adequacy of anesthesia. It is notable that, at all time points, the lidocaine group did have a trend toward higher VAS scores and a wider inter-quartile ranges than the placebo group (Table 3). Additionally, the lidocaine group experienced a twofold higher increase in pain (increase 0 to $24 \mathrm{~mm}$ vs 0 to $12 \mathrm{~mm}$ ) after catheterization as compared to the placebo group. Although the VAS has been validated for use in a urogynecological population, participants may have a variable perception of pain versus discomfort with catheterization which is not distinguished using the VAS.

Alternatively, because of the small sample size, there may have been baseline differences in perception of discomfort between groups despite randomization.

Our study had a robust double-blind placebo-controlled randomized design. This study was the first known, per a PubMed search at the time of final manuscript, to include only women and full UDS when assessing the effect of urethral anesthesia on clinically relevant urodynamic study parameters. We also used strict inclusion and exclusion criteria, aiming to exclude confounding factors such as anticholinergic crossreactivity in medications, underlying neurologic or bladder conditions that may influence bladder neurophysiology, and the presence of lower urinary tract symptoms. The use of a baseline uroflow measurement allowed us to assess whether our subjects, who reported normal voiding based on a validated questionnaire, indeed had normal voiding. Finally, allowing for physiologic filling between uroflow studies eliminated the instrumentation effect on voiding, which was previously reported. ${ }^{25}$

Our study was limited by a small sample size and did not have sufficient power to detect our primary outcome. Additionally, despite strict inclusion/exclusion criteria, our evaluation of normal voiding behaviors for inclusion only involved self-reported symptoms on the LUTS Questionnaire. A more stringent method of screening would involve screening based on baseline uroflow and VAS scores, which would have excluded the four subjects with abnormal baseline voiding characteristics (PVR $>100 \mathrm{~mL}$, elevated baseline VAS score (>50), or baseline uroflow VE <80).

Future research should focus on increasing sample size and optimizing urethral anesthesia, including dosing and administration technique. Further, a cross-over design, where participants perform UDS in each study condition on different days (to minimize catheterization effect), could reduce confounding factors by having participants serve as their own control. It is unknown if the chosen urethral anesthesia, $2 \%$ lidocaine gel, is the ideal urethral anesthetizing agent. Shafik et al. utilized 5\% xylocaine and found differences in urine flow patterns and vesical pressures during voiding; they did not report VE thus further research should investigate the impact of varying concentrations of anesthetics on VE. ${ }^{21}$ Higher concentration anesthetics, or longer incubation times, could produce a greater effect size and explain why a difference in VE was not found with $2 \%$ lidocaine in the current study. Finally, we chose to use gel rather than injectable lidocaine, as injectable lidocaine is not clinically feasible for UDS, may increase immediate pain, has a higher risk of toxicity, and placebo injections of saline have been shown to also provide an anesthesia effect. ${ }^{26}$ 
Given the impact of incomplete bladder emptying/urinary retention and the limited treatment options available, it is imperative to understand better the mechanisms contributing to inadequate voiding and whether the urethra-bladder augmentation reflex is a possible target for future therapeutics. In this randomized controlled trial of intraurethral $2 \%$ lidocaine, voiding efficiency was not decreased between groups. However, on micturition studies, a greater proportion of subjects who received lidocaine demonstrated interrupted urinary flow patterns and elevated perineal EMG activity, suggesting a role for sensory feedback during voiding. In regard to intraurethral lidocaine use during UDS, we would not recommend routine use given the impact on the voiding phase of micturition, as well as the lack of reduction in pain in the current study.

\section{Acknowledgments}

Funding supported by the Charles B. Hammond Research Fund, Duke University School of Medicine, Durham, NC (CKK) the National Institutes of Health grant K12 DK100024 (EJG), and the Pratt School of Engineering Faculty Discretionary Fund.

\section{References}

1. Fowler CJ, Griffiths D, de Groat WC. The neural control of micturition. Nature reviews Neuroscience. 2008; 9(6):453-466. [PubMed: 18490916]

2. Jung SY, Fraser MO, Ozawa H, et al. Urethral afferent nerve activity affects the micturition reflex; implication for the relationship between stress incontinence and detrusor instability. The Journal of urology. 1999; 162(1):204-212. [PubMed: 10379788]

3. Bump RC. The urethrodetrusor facilitative reflex in women: results of urethral perfusion studies. American journal of obstetrics and gynecology. 2000; 182(4):794-802. [PubMed: 10764455]

4. Gustafson KJ, Creasey GH, Grill WM. A urethral afferent mediated excitatory bladder reflex exists in humans. Neuroscience letters. 2004; 360(1-2):9-12. [PubMed: 15082166]

5. Kennelly MJ, Arena KC, Shaffer N, et al. Electrical stimulation of the urethra evokes bladder contractions in a woman with spinal cord injury. J Spinal Cord Med. 2010; 33(3):261-265. [PubMed: 20737800]

6. Kennelly MJ, Bennett ME, Grill WM, Grill JH, Boggs JW. Electrical stimulation of the urethra evokes bladder contractions and emptying in spinal cord injury men: case studies. J Spinal Cord Med. 2011; 34(3):315-321. [PubMed: 21756572]

7. Yoo PB, Horvath EE, Amundsen CL, Webster GD, Grill WM. Multiple pudendal sensory pathways reflexly modulate bladder and urethral activity in patients with spinal cord injury. The Journal of urology. 2011; 185(2):737-743. [PubMed: 21168860]

8. Peng CW, Chen JJ, Cheng CL, Grill WM. Role of pudendal afferents in voiding efficiency in the rat. American journal of physiology Regulatory, integrative and comparative physiology. 2008; 294(2):R660-672.

9. McGee MJ, Grill WM. Modeling the spinal pudendo-vesical reflex for bladder control by pudendal afferent stimulation. Journal of computational neuroscience. 2016; 40(3):283-296. [PubMed: 26968615]

10. Peng CW, Chen JJ, Cheng CL, Grill WM. Improved bladder emptying in urinary retention by electrical stimulation of pudendal afferents. Journal of neural engineering. 2008; 5(2):144-154. [PubMed: 18430976]

11. Coyne KS, Zhou Z, Thompson C, Versi E. The impact on health-related quality of life of stress, urge and mixed urinary incontinence. BJU international. 2003; 92(7):731-735. [PubMed: 14616456]

12. Richter HE, Nygaard I, Burgio KL, et al. Lower urinary tract symptoms, quality of life and pelvic organ prolapse: irritative bladder and obstructive voiding symptoms in women planning to undergo 
abdominal sacrocolpopexy for advanced pelvic organ prolapse. The Journal of urology. 2007; 178(3 Pt 1):965-969. [PubMed: 17632167]

13. Stoffel, J; Lightner, D; Peterson, A; Sandhu, J; Suskind, A; Wei, J. Non-neurogenic chronic urinary retention: consensus definition, management strategies, and future opportunities. 2014. https:// www.auanet.org/guidelines/chronic-urinary-retention. Accessed April 19, 2017

14. Coyne KS, Barsdorf AI, Thompson C, et al. Moving towards a comprehensive assessment of lower urinary tract symptoms (LUTS). Neurourology and urodynamics. 2012; 31(4):448-454. [PubMed: 22396308]

15. Danforth KN, Townsend MK, Lifford K, Curhan GC, Resnick NM, Grodstein F. Risk factors for urinary incontinence among middle-aged women. Am J Obstet Gynecol. 2006; 194(2):339-345. [PubMed: 16458626]

16. Rosier PF, Schaefer W, Lose G, et al. International Continence Society Good Urodynamic Practices and Terms 2016 Urodynamics, uroflowmetry, cystometry, and pressure-flow study. Neurourology and urodynamics. 2016

17. Lukacz ES, Lawrence JM, Burchette RJ, Luber KM, Nager CW, Buckwalter JG. The use of Visual Analog Scale in urogynecologic research: a psychometric evaluation. Am J Obstet Gynecol. 2004; 191(1):165-170. [PubMed: 15295359]

18. Lee WC, Wu CC, Wu HP, Tai TY. Lower urinary tract symptoms and uroflowmetry in women with type 2 diabetes mellitus with and without bladder dysfunction. Urology. 2007; 69(4):685-690. [PubMed: 17445652]

19. Nishizawa O, Satoh S, Harada T, et al. Role of the pudendal nerves on the dynamics of micturition in the dog evaluated by pressure flow EMG and pressure flow plot studies. The Journal of urology. 1984; 132(5):1036-1039. [PubMed: 6492271]

20. Peng CW, Chen JJ, Chang HY, de Groat WC, Cheng CL. External urethral sphincter activity in a rat model of pudendal nerve injury. Neurourology and urodynamics. 2006; 25(4):388-396. [PubMed: 16637068]

21. Shafik A, Shafik AA, El-Sibai O, Ahmed I. Role of positive urethrovesical feedback in vesical evacuation. The concept of a second micturition reflex: the urethrovesical reflex. World journal of urology. 2003; 21(3):167-170. [PubMed: 12898170]

22. Stav K, Ohlgisser R, Siegel YI, Beberashvili I, Padoa A, Zisman A. Pain during female urethral catheterization: intraurethral lubricant injection versus catheter tip lubrication-A prospective randomized trial. The Journal of urology. 2015; 194(4):1018-1021. [PubMed: 25818032]

23. Chan MF, Tan HY, Lian X, et al. A randomized controlled study to compare the $2 \%$ lignocaine and aqueous lubricating gels for female urethral catheterization. Pain practice : the official journal of World Institute of Pain. 2014; 14(2):140-145. [PubMed: 23560476]

24. Todd KH, Funk KG, Funk JP, Bonacci R. Clinical significance of reported changes in pain severity. Ann Emerg Med. 1996; 27(4):485-489. [PubMed: 8604867]

25. Suskind AM, Smith PP. Evidence of a functional effect of transient transurethral catheterization on micturition in women. International urogynecology journal. 2012; 23(9):1245-1248. [PubMed: 22249279]

26. Miller L, Jensen MP, Stenchever MA. A double-blind randomized comparison of lidocaine and saline for cervical anesthesia. Obstetrics and gynecology. 1996; 87(4):600-604. [PubMed: 8602315] 


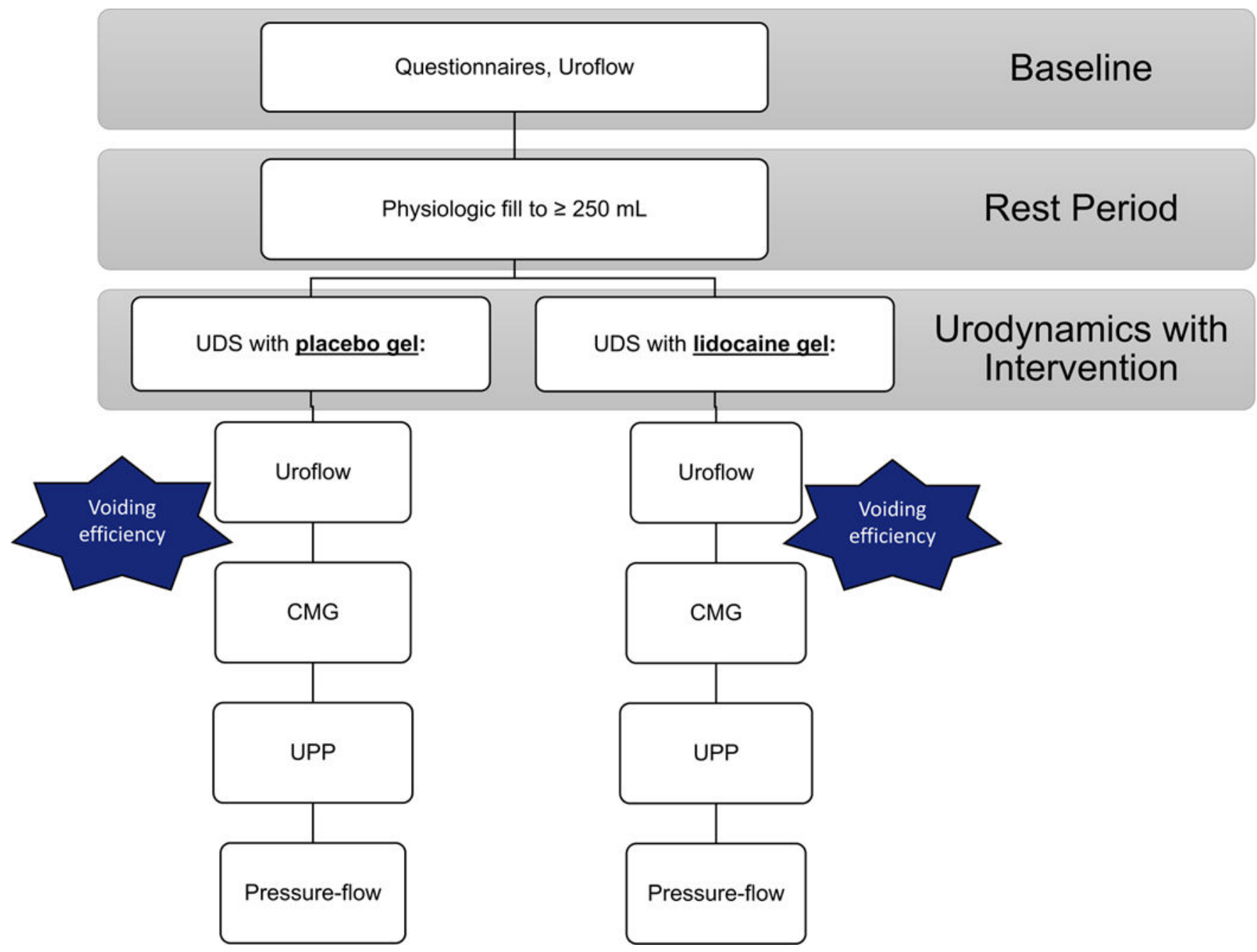

Figure 1.

Methods Flow Diagram. BS, bladder scanner; LUTS, Lower Urinary Tract Symptom Questionnaire; PVR, post void residual; UDS, urodynamic study; UPP, urethral pressure profilometry. 




Figure 2.

Study Flow Diagram. LUTS, Lower Urinary Tract Symptom. *17 subjects were excluded: 14 declined after being informed, in detail, of the study protocol, 3 were excluded due to meeting exclusion criteria (BMI >40, self-reported LUTS). 


\section{Table 1}

Baseline Demographics. ARS, anticholinergic risk scale; BMI, body mass index; FCI, functional comorbidity index; LUTS, lower urinary tract symptom; POP, pelvic organ prolapse. Data are presented as mean \pm standard deviation, median [IQR], or $\mathrm{n}(\%)$.

\begin{tabular}{|c|c|c|}
\hline Variable & $\begin{array}{l}\text { Placebo } \\
(n=12)\end{array}$ & $\begin{array}{l}\text { Lidocaine } \\
(n=11)\end{array}$ \\
\hline Age (y) & $33 \pm 9.2$ & $30 \pm 9.6$ \\
\hline \multicolumn{3}{|l|}{ Race } \\
\hline White & $6(50.0)$ & $6(54.5)$ \\
\hline Black & $6(50.0)$ & $3(27.3)$ \\
\hline Asian & $0(0)$ & $2(18.2)$ \\
\hline $\mathrm{BMI}\left(\mathrm{kg} / \mathrm{m}^{2}\right)$ & $25 \pm 5.0$ & $23 \pm 3.7$ \\
\hline \multicolumn{3}{|l|}{ Education } \\
\hline High school & $0(0)$ & $0(0)$ \\
\hline Associate & $3(25.0)$ & $0(0)$ \\
\hline College graduate & $3(25.0)$ & $5(45.5)$ \\
\hline Graduate/professional & $6(50.0)$ & $6(54.5)$ \\
\hline \multicolumn{3}{|l|}{ Mode of Delivery } \\
\hline Vaginal delivery & $4(33.3)$ & $2(18.2)$ \\
\hline Cesarean section & $2(16.7)$ & $0(0)$ \\
\hline \multicolumn{3}{|l|}{ Menopausal status } \\
\hline Premenopausal & $11(91.7)$ & $10(90.9)$ \\
\hline Postmenopausal & $1(8.33)$ & $1(9.09)$ \\
\hline Smoker & $0(0)$ & $0(0)$ \\
\hline POP & $0(0)$ & $0(0)$ \\
\hline ARS score & $0(0,0)$ & $0(0,0)$ \\
\hline FCI & $0(0,0)$ & $0(0,0)$ \\
\hline LUTS Questionnaire score & $2.5[1.25,4]$ & $5[3,7]$ \\
\hline
\end{tabular}

Female Pelvic Med Reconstr Surg. Author manuscript; available in PMC 2020 July 01. 


\section{Table 2}

Uroflowmetry, Cystometry, and Micturition Pressure-Flow Study Data. Voiding efficiency $=$ (voided volume / (voided volume + residual volume) $\times 100$ ); DO, detrusor overactivity; EMG, electromyography; MCC, maximum cystometric capacity; MUCP, maximum urethral closure pressure; Pabd, abdominal pressure; Pdet, detrusor pressure; PVR, post void residual; Qmax, maximum flow rate. Data are presented as mean \pm standard deviation, median [IQR], or n (\%). Analyses were performed by $\chi^{2}$ for categorical values, Student $t$ test for continuous variables, or Mann-Whitney $\mathrm{U}$ when non-parametric tests were indicated.

\begin{tabular}{|c|c|c|c|c|c|c|c|}
\hline \multicolumn{8}{|c|}{ Uroflowmetry } \\
\hline \multicolumn{3}{|l|}{ Variable } & \multicolumn{2}{|c|}{ Placebo $(n=12)$} & \multicolumn{2}{|c|}{ Lidocaine (n=11) } & $\mathbf{P}^{*}$ \\
\hline \multicolumn{3}{|c|}{ Baseline voiding efficiency $(\%)$} & \multicolumn{2}{|c|}{$92.8 \pm 5.14$} & \multicolumn{2}{|c|}{$91.3 \pm 7.51$} & 0.58 \\
\hline \multicolumn{3}{|c|}{ Post-intervention voiding efficiency (\%) } & \multicolumn{2}{|c|}{$89.3[85.9,93.9]$} & \multicolumn{2}{|c|}{$89.5[82.5,91.7]$} & 0.74 \\
\hline \multicolumn{8}{|l|}{ Baseline } \\
\hline \multicolumn{3}{|c|}{ Average flow rate $(\mathrm{mL} / \mathrm{s})$} & \multicolumn{2}{|c|}{$17.1 \pm 7.46$} & \multicolumn{2}{|c|}{$16.9 \pm 3.85$} & 0.95 \\
\hline \multicolumn{3}{|c|}{ Maximum flow rate (mL/s) } & \multicolumn{2}{|c|}{$35.2 \pm 16.1$} & \multicolumn{2}{|c|}{$30.3 \pm 8.18$} & 0.40 \\
\hline \multicolumn{3}{|l|}{ Flow time (s) } & \multicolumn{2}{|c|}{$24.3[15.6,57.9]$} & \multicolumn{2}{|c|}{$23.6[20.0,35.1]$} & 1.00 \\
\hline \multicolumn{8}{|l|}{ Post-intervention } \\
\hline \multicolumn{3}{|c|}{ Average flow rate $(\mathrm{mL} / \mathrm{s})$} & \multicolumn{2}{|c|}{$16.3 \pm 6.28$} & \multicolumn{2}{|c|}{$12.9 \pm 4.53$} & 0.15 \\
\hline \multicolumn{3}{|c|}{ Maximum flow rate (mL/s) } & \multicolumn{2}{|c|}{$36.5 \pm 14.4$} & \multicolumn{2}{|c|}{$26.4 \pm 9.87$} & 0.07 \\
\hline \multicolumn{3}{|l|}{ Flow time (s) } & \multicolumn{2}{|c|}{$39.9[21.1,57.5]$} & \multicolumn{2}{|c|}{$41.5[27.3,63.7]$} & 0.61 \\
\hline \multicolumn{8}{|c|}{ Cystometry } \\
\hline Variable & Placeb & $(n=12)$ & Lidoca & ne $(n=11)$ & $\mathbf{P}^{*}$ & & \\
\hline First sensation (mL) & $51.0[3$ & $.0,69.0]$ & $61.0[3$ & $.0,102]$ & 0.70 & & \\
\hline First desire (mL) & $97.0[7$ & $.8,192]$ & $111[76$ & $0,208]$ & 0.92 & & \\
\hline Strong desire $(\mathrm{mL})$ & $343 \pm$ & & $309 \pm 1$ & & 0.64 & & \\
\hline $\mathrm{MCC}(\mathrm{mL})$ & $421 \pm$ & & $387 \pm 1$ & & 0.65 & & \\
\hline DO & $1(8)$ & & $1(9)$ & & 0.95 & & \\
\hline Incontinence & $1(8)$ & & $0(0)$ & & 0.33 & & \\
\hline $\mathrm{MUCP}\left(\mathrm{cmH}_{2} \mathrm{O}\right)$ & $142 \pm 3$ & 7.4 & $159 \pm 3$ & 8.6 & 0.30 & & \\
\hline & & Pressure & Flow & & & & \\
\hline Variable & & Placebo & $(n=12)$ & Lidocain & $e(n=11)$ & $\mathbf{P}^{*}$ & \\
\hline PVR (mL) & & $45[26.3$ & $58.8]$ & $50.0[30.0$ & $0,150]$ & 0.35 & \\
\hline Maximum Pdet $(\mathrm{cmH}$ & O) & $65.1 \pm 3$ & & $60.2 \pm 30$ & & 0.74 & \\
\hline Pdet@Qmax $\left(\mathrm{cmH}_{2} \mathrm{O}\right.$ & & $31.0 \pm 1$ & & $37.6 \pm 19$ & & 0.42 & \\
\hline Maximum Pabd (cmH & $\left.{ }_{2} \mathrm{O}\right)$ & $38.9 \pm 1$ & & $43.5 \pm 26$ & & 0.62 & \\
\hline Flow time (s) & & $46.5 \pm 1$ & & $55.6 \pm 29$ & & 0.38 & \\
\hline Pabd recruitment duri & ig flow & $1(8)$ & & $5(46)$ & & 0.07 & \\
\hline
\end{tabular}

Female Pelvic Med Reconstr Surg. Author manuscript; available in PMC 2020 July 01. 


\begin{tabular}{llll}
\hline \multicolumn{4}{c}{ Pressure-Flow } \\
\hline Variable & Placebo (n=12) & Lidocaine (n=11) & $\mathbf{P}^{*}$ \\
\hline Interrupted flow curve & $0(0)$ & $4(36)$ & $0.02^{*}$ \\
Elevated EMG activity & $3(25)$ & $8(73)$ & $0.02^{*}$ \\
\hline Voiding efficiency $(\%)$ & $92.2[90.1,95.0]$ & $89.8[80.7,92.1]$ & 0.13 \\
\hline
\end{tabular}

p $\leq 0.05$ was considered statistically significant. 
Table 3

Visual Analog Scale (VAS) Scores. MCC, maximum cystometric capacity. Data are presented as median [IQR]. Analyses were performed by the Mann-Whitney U test.

\begin{tabular}{llll}
\hline Variable & $\begin{array}{l}\text { Placebo } \\
(\mathbf{n = 1 2})\end{array}$ & $\begin{array}{l}\text { Lidocaine } \\
(\mathbf{n = 1 1})\end{array}$ & $\mathbf{P}^{*}$ \\
\hline Baseline $(\mathrm{mm})$ & $0.0[0.0-0.8]$ & $0.0[0.0-2.0]$ & 0.45 \\
Catheterization $(\mathrm{mm})$ & $12[3.0,29]$ & $24[12,41]$ & 0.35 \\
$100 \mathrm{~mL}$ filling $(\mathrm{mm})$ & $0.0[0.0,2.0]$ & $1.0[0.0,7.0]$ & 0.35 \\
MCC $(\mathrm{mm})$ & $5.0[0.0,14]$ & $11[1.0,18]$ & 0.53 \\
\hline
\end{tabular}

p $\leq 0.05$ was considered statistically significant. 